\title{
Two Greens; A New Signal Aspect for High-Speed Train on Wayside Signaling
}

\author{
Hiroshi UGAJIN \\ Senior Researcher \\ Fundamental Research Dept., RTRI \\ Hiroaki SHIROTO \\ Researcher \\ Koji OMINO \\ Researcher \\ Kohei FUJINAMI \\ Researcher
}

Prior to the practical use of a new wayside signal aspect for high-speed trains in the Hokuhoku Line, its visibility and compatibility were tested about the signaling method which is of a double green aspect given before the appearance of a single green aspect for the proceed signal. Twenty four test runs were thoroughly performed with participation of twelve drivers, on the line of $30 \mathrm{~km}$ at the maximum speed of $160 \mathrm{~km} / \mathrm{h}$. Drivers succeeded in identifying without difficulty signals given by the new system for each of test runs.

Keywords : wayside signaling, high-speed train, signal aspect, signal visibility, ergonomics

\section{Introduction}

March 22, 1997, the Hokuetsu Express, "Hokuhoku" Line of $59.5 \mathrm{~km}$ opened service as a shortcut between the Muikamachi Station of Joetsu Line and Saigata Station of Shin-etsu Line. On this line, the train runs at $150 \mathrm{~km} / \mathrm{h}$, the highest in Japan for the narrow gauge railway with wayside signaling. This is a line designed on the basis of highlevel standard, allowing speed increase to $160 \mathrm{~km} / \mathrm{h}$ in the near future. In order to achieve such high-speed operation, there were various technical subjects to overcome, one of which was concerned with the signaling method.

The highest train speed with the conventional color light signal has been $130 \mathrm{~km} / \mathrm{h}$ which is barely attained by the operation according to the signal aspect. In Japan, a driver has to fix his eyes on and recognize a signal at an entry into a block section while still at a point about $600 \mathrm{~m}$ before the signal, controlling the speed to the signaled level before reaching the signal point. Signal aspect stages and standard directing speed on main lines are shown in Table 1. As is shown in the table, the driver running a train at $130 \mathrm{~km} / \mathrm{h}$ has to decelerate the speed to $75 \mathrm{~km} / \mathrm{h}$ when observing an aspect of lower signal in the next section. This is the limit attainable with the existing block section length and vehicle's deceleration performance.

In order to achieve the high-speed train operation at $130-160 \mathrm{~km} / \mathrm{h}$ with the above signaling system and running conditions, there is no way but to increase the number of signal aspect stages. Discussions have been made since around 1980 concerning which type of signal method is appropriate for this purpose. Consequently, in the Hokuhoku

Table 1 Signal aspects and train speed on main lines (sample)

\begin{tabular}{l|lc|c}
\hline \multicolumn{1}{c|}{ Name } & \multicolumn{2}{|c}{ Aspect } & Directed Speed \\
\hline Proceed signal & Green & $(\mathrm{G})$ & $130 \mathrm{~km} / \mathrm{h}$ \\
\hline Reduced speed signal & Yellow and Green & $(\mathrm{YG})$ & $75 \mathrm{~km} / \mathrm{h}$ \\
\hline Caution signal & Yellow & $(\mathrm{Y})$ & $45 \mathrm{~km} / \mathrm{h}$ \\
\hline Restricted speed signal & Two Yellows & $(\mathrm{YY})$ & $25 \mathrm{~km} / \mathrm{h}$ \\
\hline Stop signal & Red & $(\mathrm{R})$ & $0 \mathrm{~km} / \mathrm{h}$ \\
\hline
\end{tabular}

Line (Hokuetsu-Hoku Line as referred to in the construction project), the new aspect compatible with high-speed operation was added to the existing signal aspect system. This new aspect is the signal aspect with two green lights (speedup signal or GG) and located on the higher level side of proceed signal. Namely, this is a clear signal to allow the train which can be operated at $130 \mathrm{~km} / \mathrm{h}$ or higher to proceed at the allowable maximum speed. In this case, the $\mathrm{G}$ signal functions as a restricted proceed signal to allow the train to proceed at $130 \mathrm{~km} / \mathrm{h}$ or less.

A problem associated with practical application of the new signal system is the visibility of the signal. To ensure safe and smooth operation, it was essential to verify the visibility of the GG signal in the course of a series of operations. In other words, it was necessary to confirm that a driver can discriminate the new aspect from other aspects without loosing his work allowance during operation.

Various examinations necessary for authorization of the Hokuetsu-Hoku Line were conducted from September to October, 1996, and the signal visibility examination was made in the latter half of the period. This paper introduces details up to implementation of the examination and reports the results of the examinations concerned.

\section{Wayside signaling for high-speed train}

\section{1 Particulars of new signal aspects}

For the $160 \mathrm{~km} / \mathrm{h}$ operation on the local line, it was proposed to employ partially or wholly the cab signal. How ${ }^{-}$ ever, in a relatively early time, the proposition of using current wayside signaling gained much support in view of the necessity of achieving speed-up with low costs while utilizing existing wayside facilities. Besides, available signal colors were restricted to Green, Yellow and Red. Considering existing aspects, therefore, there were only two options for new aspect; GG or a combination of certain flickering signal patterns. With this background, the study on visibility of these new aspect plans was started early in line with 
the study on the signaling method ${ }^{1)}$.

As regards signaling, a dispute on where the new aspect should be positioned in the existing system could not be determined readily. Particularly important is a fact that the exiting $G$ signal becomes a restricted proceed signal, though only for certain trains or sections, when the new aspect is positioned on the higher level side of the aspect system. For the driver to operate the train under the proposed signal system, this is not a simple change in reading of directing speed of the signal, which has been made up to now. Rather, this means that the common cognitive framework must be replaced by a special one, requiring certain assistance for the driver. For example, it was proposed to provide a signal aspect ( $\mathrm{G}$ flickering) to the section before the $\mathrm{G}$ aspect section or to transmit an advance alarm to the vehicle. After all, it was decided not to provide any special measure on the condition that the ATS-P system will function as a backup for the stop signal.

\section{2 Visibility examination of two greens}

A full-scale examination on basic visibility of GG signal was made in 1988. This visibility was compared with existing five aspects through ground and running observation examinations. The result verified the satisfactory visibility of GG signal at a specified confirmation distance of $600 \mathrm{~m}$. The examination also involved comparison with the GG alternate flashing aspect in addition to the GG signal, with extremely satisfactory results. Because of the uncomfortable feeling of the driver when reading the flashing signal and the magnitude of concern of possible misidentification, however, it was decided to exclude the flashing signal from the scope of discussion in the future.

According to the examination result, there was a high possibility that remote observation of two green (GG) lamps spaced with a center-to-center distance with two lamps in between (lamp distance $660 \mathrm{~mm}$ on centers) for YG and YY leads to misidentification of two lamps as "G" because they are visually merged. It was therefore proposed to separate two green lamps by a distance for three lamps (center distance of lamps, $880 \mathrm{~mm}$ ) to eliminate such possibility. The distance separating two lamps at a visual range of $600 \mathrm{~m}$ may be expressed as follows in terms of the visual angle; 2'55" for the distance equivalent to two intermediate lamps and 4'11" for the distance equivalent to three intermediate lamps.

It was decided to confirm the visibility of GG signal with the extended lamp distance in the Kosei Line speedup examination in 1990 . The study was also made to see if the driver could identify the signal without undue attention during running at $160 \mathrm{~km} / \mathrm{h}$. Namely, under the condition that GG was indicated on all signals other than the one which indicated $\mathrm{G}$, each of four drivers performed three test runs. In all examinations, drivers could confirm the signal correctly before reaching the specified position. In this way, practical application of the GG signal could be expected ${ }^{2}$.

\section{3 Repeating system for two greens}

For practical application of the GG signal, the study on a repeating signal system responding to the new signal as-

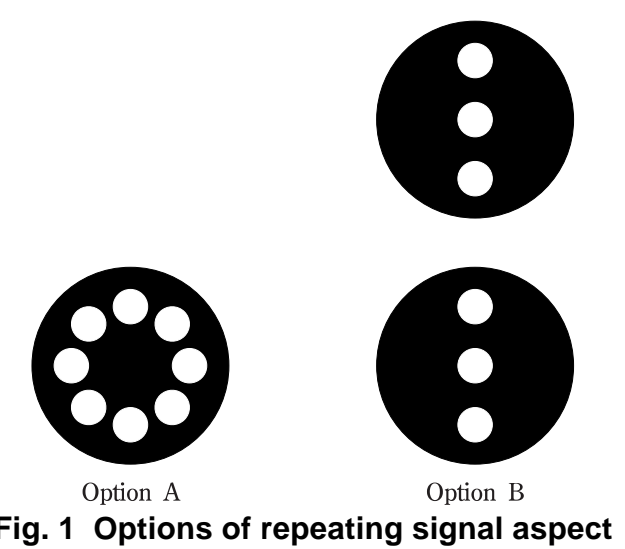

pect system is essential. Researches and examinations have been made on this problem since 1991. Similarly to the conception of GG signal, establishing its responding repeating signal aspect in addition with the existing position light signal repeating system became a prospective project. For the new signal aspect, propositions were narrowed down to two options as shown in Fig. 1. But the final decision on which option to chose was postponed.

In 1996, it became urgently necessary to decide the signal aspect option in view of the signal equipment work of the Hokuetsu-Hoku Line. The running observation examination on the open section had already been completed for two options, but its result was not enough to determine their merits and demerits. This time, it was decided to determine the method to be employed for this Line on the basis of comparative research on the signal visibility in a tunnel with a prototype tunnel signal mechanism.

In this examination, 24 test subjects (drivers or those who have once been a driver) attempted identification of each aspect option three times at three visual distances (200, 240 , and $280 \mathrm{~m}$ ) with and without lighting in the tunnel. The subjects were requested to add if they were confident or not at a time of judgment. The result showed 100\% percentage of correct which means that merits and demerits of both options could not be determined from this result. Then, the percentage of definite answer or the ratio of "confirmative answers" was calculated for the same test conditions ( 24 subjects $\times 3$ times $=72$ cases) so as to be used as a visibility index. These indices were compared for

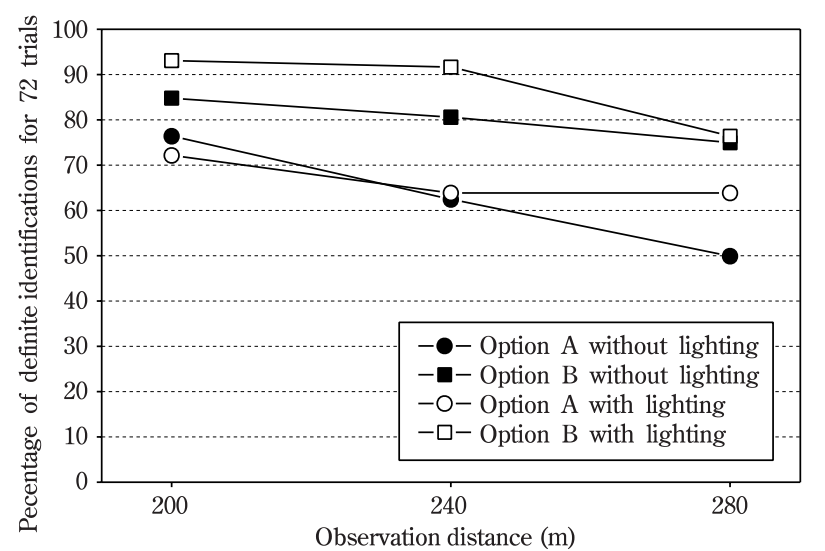

Fig. 2 Results of repeating signal identification in the tunnel 
respective test conditions (Fig. 2). In consequence, it was statistically verified that the option $B$ is superior in visibility under four types of conditions excluding cases of $280 \mathrm{~m}$ with lighting and $200 \mathrm{~m}$ without lighting. After all, it was planned to proceed with preparation for employment of the option B on the Hokuetsu-Hoku Line.

\section{Examination of signal visibility in Hokuetsu-Hoku Line}

\section{1 Purpose}

The purpose of this examination was to confirm in terms of visibility and workability the safety of the highspeed signal which is introduced for the high-speed operation at $160 \mathrm{~km} / \mathrm{h}$ in Hokuetsu-Hoku Line. To achieve this difficult purpose under restricted test conditions, the minute examination plan and careful preparation were made.

\section{2 Method}

\section{2. 1 Target signals along the test section}

The examination was made on the down track of about $30 \mathrm{~km}$ from the Matsudai Station in the middle $(29 \mathrm{k} 166 \mathrm{~m} 67)$ of the Line to a terminal station called Saigata Station (59k467m80). There were 24 signals ( 17 main independent and 7 repeating signals) from the departure signal at Matsudai to the home signal at Saigata, excluding the one on each end of the section. The examination was made on these signals (Table 2). Note that Nos. 20 and 24 signals are those with the proceed signal as the highest-level aspect. Accordingly, as signals with high speed signal aspect, there are 15 main signals and 3 repeating signals. These signals are illustrated in Fig. 3.

\section{2. 2 Offered train and drivers}

The offered train consisted of $2 \mathrm{M} 3 \mathrm{~T}$ of HK-681 series vehicles (reformed makeup for high-speed examination). Four test runs per day during daytime span (9:27 - 15:47) were planned. The train speed in the test section was the maximum possible $(160 \mathrm{~km} / \mathrm{h}$ at the highest) on the train performance curve regardless of the signal aspect.

Twelve drivers belonging to the East Japan Railway

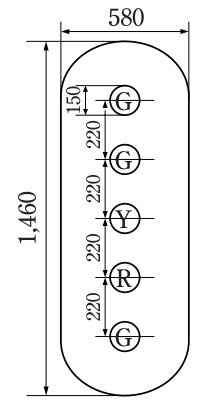

(a) Independent signal

(5 lights)

for R, Y, G, GG

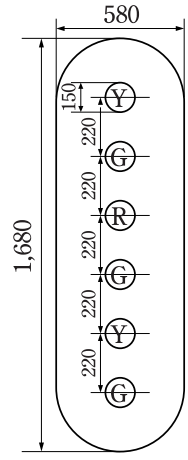

(b) Independent signal (6 lights)

for R, Y, YG, G, GG

Company participated in operation of the offered train. According to the schedule, two drivers operated the train alternately in one day (one driver to operate the train twice) and the test run was completed in six days. They were also test subjects of the signal visibility examination. Four subjects were selected from 30, 40, and 50-year groups respectively.

\section{2. 3 Condition of signal aspects}

Unless any measure was taken, all signals for examination were to indicate the highest level signal for the offered train. Of main 15 signals with the speed-up signal aspect, 13 signals excluding Nos. 4 and 21 were provided with a temporary means to control the highest level aspect to the proceed signal as required. With 13 units of control,

Table 2 Signals to be tested and their visibility conditions

\begin{tabular}{|c|c|c|c|c|c|c|}
\hline \multicolumn{2}{|c|}{ Signals } & \multicolumn{2}{|c|}{ Position } & \multicolumn{2}{|c|}{ Signal calling point } & \multirow{2}{*}{$\begin{array}{l}\text { Observation } \\
\text { distance }(\mathrm{m})\end{array}$} \\
\hline No. & type & kilometer & lighting & kilometer & lighting & \\
\hline 1 & $\mathrm{BS}$ & 31,585 & $\mathrm{D}$ & 30,903 & $\mathrm{D}$ & 682 \\
\hline 2 & $\mathrm{BS}$ & 32,495 & $\mathrm{D}$ & 31,772 & $\mathrm{D}$ & 723 \\
\hline 3 & $\mathrm{HS}$ & 33,781 & $\mathrm{D}$ & 33,058 & $\mathrm{D}$ & 723 \\
\hline 4 & DS & 34,263 & $\mathrm{D}$ & 34,005 & $\mathrm{D}$ & 258 \\
\hline 5 & $\mathrm{BS}$ & 36,505 & $\mathrm{D}$ & 35,782 & $\mathrm{D}$ & 723 \\
\hline 6 & $\mathrm{BS}$ & 38,263 & $\mathrm{D}$ & 37,540 & $\mathrm{D}$ & 723 \\
\hline 7 & $\mathrm{BS}$ & 42,247 & $\mathrm{D}$ & 41,499 & $\mathrm{D}$ & 748 \\
\hline 8 & BS & 43,306 & $\mathrm{D}$ & 42,558 & $\mathrm{D}$ & 748 \\
\hline 9 & $\mathrm{RS}$ & 43,881 & $\mathrm{D}$ & 43,681 & $\mathrm{D}$ & 200 \\
\hline 10 & $\mathrm{HS}$ & 44,527 & $\mathrm{~L}$ & 43,881 & $\mathrm{D}$ & 646 \\
\hline 11 & DS & 44,968 & $\mathrm{~L}$ & 44,368 & $\mathrm{~L}$ & 600 \\
\hline 12 & $\mathrm{BS}$ & 47,758 & $\mathrm{~L}$ & 47,030 & $\mathrm{~L}$ & 728 \\
\hline 13 & $\mathrm{BS}$ & 50,191 & $\mathrm{D}$ & 49,505 & $\mathrm{D}$ & 686 \\
\hline 14 & $\mathrm{RS}$ & 51,710 & $\mathrm{~L}$ & 51,471 & $\mathrm{D}$ & 239 \\
\hline 15 & $\mathrm{RS}$ & 51,931 & $\mathrm{~L}$ & 51,710 & $\mathrm{~L}$ & 221 \\
\hline 16 & $\mathrm{BS}$ & 52,188 & $\mathrm{~L}$ & 51,931 & $\mathrm{~L}$ & 257 \\
\hline 17 & $\mathrm{RS}$ & 52,680 & $\mathrm{~L}$ & 52,507 & $\mathrm{~L}$ & 173 \\
\hline 18 & $\mathrm{RS}$ & 52,898 & $\mathrm{D}$ & 52,680 & $\mathrm{~L}$ & 218 \\
\hline 19 & $\mathrm{RS}$ & 53,022 & $\mathrm{D}$ & 52,898 & $\mathrm{D}$ & 124 \\
\hline 20 & $\mathrm{HS}$ & 53,202 & $\mathrm{~L}$ & 53,022 & D & 180 \\
\hline 21 & DS & 53,656 & $\mathrm{~L}$ & 53,406 & $\mathrm{~L}$ & 250 \\
\hline 22 & $\mathrm{BS}$ & 54,915 & $\mathrm{~L}$ & 54,219 & $\mathrm{~L}$ & 696 \\
\hline 23 & $\mathrm{RS}$ & 56,400 & $\mathrm{~L}$ & 56,161 & $\mathrm{~L}$ & 239 \\
\hline 24 & $\mathrm{BS}$ & 56,858 & $\mathrm{~L}$ & 56,400 & $\mathrm{~L}$ & 458 \\
\hline
\end{tabular}

Type BS: Blocking Signal, HS: Home Signal, DS: Departure Signal, RS: Repeating Signal

Lighting L: Light in the open area, D: Dark in the tunnel area

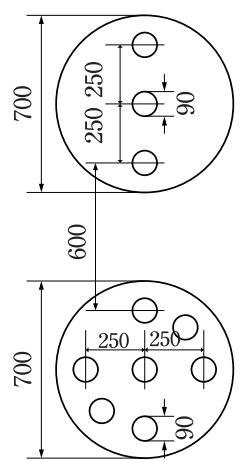

(c) Repeating signal for the open are

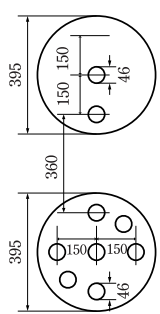

(d) Repeating signal for the tunnel area

Fig. 3 Summary of signaling mechanisms 
random setting was made, with due consideration of test conditions, so that the signal aspect during test should become the proceed signal or speed-up signal. Repeating signals were planned to repeat the aspects of main signals.

These signal aspect conditions were informed neither to drivers nor to others except for those directly concerned.

On the examination day, control of signal aspects was made entirely from the Muikamachi CTC center.

\section{2. 4 Examination Procedure}

The examination was made for six days from October 9 to $14,1996$.

Each driver was provided with an orientation concerning details of the examination and engaged in operation of the offered train during the speed-up test, etc. beginning in September 28. Alternatively, they took part in the examination after having attended the handle training implemented from October 6 to 8. During the examination, drivers operated the offered train under operation instructions as required. In the course of operation, they were requested to perform signal calling at a specified position for the proceed signal or speed-up signal of 24 signals including seven repeating signals.

The behavior and voice of drivers during operation were recorded on videotape, together with the image with voice as viewed from the right side, foreground image, and elapsed time. Vehicle side signals (tachometer-generator pulse, change of notch on powering or braking) were sampled by the operating data recording system for No.681 series car and recorded, as every second data, in a floppy disk, together with calculation results of the speed and running distance. Simultaneously, they were output as analog signals to the data recorder. Along with these data, the voice waveform of signal calling was recorded in this recorder.

\section{3 Results}

\section{3. 1 Estimation of observed distance at signal calling}

To estimate the observed distance at signal calling, the calculation error relative to the actual distance was corrected for the distance pulse output (100 m interval) on the data recorder output chart, followed by determination of the estimated kilometers (at the driver head). Then, the kilometers and speed at a signal-calling time point identified on the voice waveform on the chart was determined through linear interpolation from the estimated kilometer and speed at the distance pulses before and after the above time point. The estimated error of the resulting signal-calling position (kilometers) was $\pm 5 \mathrm{~m}$.

The signal-calling time point was an instant when a driver called the type of aspect ("Shinko !" for the proceed signal or "Kosoku-Shinko!" for the speed-up signal) after the signal name, and marked while listening to the voice in the test. If there was any ambiguity concerning the content of signal calling, the video tape was played back to check the behavior and voice content of a driver and the signalcalling time point was corrected as required.

The calling position and the speed which were estimated for each signal, and confirmed signal calling content, etc. were analyzed as follows.

\section{3. 2 Performance of signal calling}

(1) Signal calling error

Of a total of 576 ( 12 drivers $\times 24$ units $\times 2$ times $)$ signal callings, correct calling was achieved in one try on approaching the specified position in 546 cases. Remaining 30 cases included incorrect initial callings or callings made after passing the specified position (in three seconds or more from the specified position).

Thirty cases include 14 failures for the proceed signal (eight case at $150 \mathrm{~km} / \mathrm{h}$ or more), 10 failures for the speedup signal (six cases at $150 \mathrm{~km} / \mathrm{h}$ or more), five failures for the repeating proceed signal (two cases at $150 \mathrm{~km} / \mathrm{h}$ or more), and one failure for the repeating speed-up signal (one case at $150 \mathrm{~km} / \mathrm{h}$ or more). All of these cases were confirmed to be attributable to the equipment-related problem and insufficient learning of drivers.

(2) Timing of signal calling

As an index for timing of signal calling, the relative calling point was taken and subjected to statistical processing, which is a distance between the specified calling position and the estimated position where the aspect is actually called, with " + " provided for the internal estimated position and " - " for the external estimated position. In this case, a total of 46 following data which were judged to be inappropriate for batch processing were excluded from the scope of statistical processing. As a result, the statistical processing was made for 530 cases.

(a) 25 cases, determined by subtracting five cases in which the calling content was corrected during a series of signal calling operations and in which no calling delay existed from 30 cases described in (1) above.

(b) 24 cases related to No.22 signal (of which three cases overlap with (a) above). This signal was reconstructed in the course of test because of faulty installation method of the signal calling position sign. Besides, installation of a repeating signal was judged necessary because the curve made visibility of the signal difficult.

Concerning the signal calling for the main signal, comparison was made among drivers by the aspect type and train speed range, using the relative distance of calling as an index. The train speeds, after reviewed from their distribution, were classified into higher and lower speed ranges

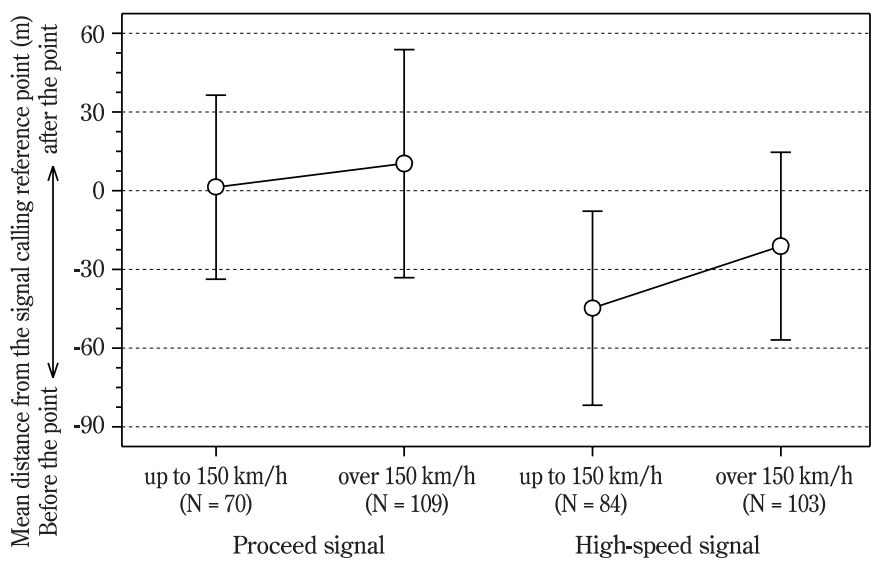

Fig. 4 Comparison of timings for signal calling per signaling type and per speed area 
with the boundary of $150 \mathrm{~km} / \mathrm{h}$. Following points were known from the processing results.

(a) The difference by aspect is 10 of 12 and thus significant. In all cases, calling for the speed-up aspect was earlier than that for the proceed aspect. (Fig. 4)

(b) Difference by speed could not be observed, excluding two of 12 subjects.

(c) Difference in variance by aspect was not observed for all drivers.

(d) Variance difference by speed was not observed except for two of 12 subjects.

With regard to calling for the repeating signal, there were only two cases where one of 12 subjects called the repeating speed-up signal while the train was running in the low-speed range. Accordingly, comparison was made only in terms of the aspect type. As a result of this processing, no difference was observed in the calling time point and its variance between proceed and speed-up signals for all subjects, except for one of 12 subjects.

On the other hand, comparison was made on relative distance of calling by the speed range for a combination of main and repeating signals. Except for one subject, the remaining 11 subjects showed no difference in the calling time points and variance between high and low speed ranges.

\section{4 Discussion}

The average of relative distance of calling may be considered as an index of signal visibility. The index decreases with increase in the visibility length. On the other hand, variance in relative distance of calling may be considered as an index of work allowance. With decreasing index, the calling timing is judged to be more stable.

Judging from the result of statistical processing of relative distance of calling, the speed-up signal is superior to the proceed signal in terms of signal visibility regardless of the train speed. As regards the repeating signal, no difference attributable to the type of aspect was observed.

Concerning the work allowance, any constant trend was not found in terms of the type of signal and aspect and the train speed. Variance in relative distance of calling showed remarkable difference among individuals, which may be attributed to the learning level of drivers. Analyzing variance of relative distance of calling for all signals by each driver shows the standard deviation of $\pm 19.9 \mathrm{~m}$ to \pm 66.4 $\mathrm{m}$. Two subjects having the highest deviation were those who have not attended the handle training for this exami- nation. This indicates that the learning contributes to stabilization of the calling timing. The questionnaire conducted with drivers after the examination contained many replies concerning unfamiliarity with the vehicles, railways, and signals.

\section{Concluding Remarks}

Of 530 signal calling time data obtained from the signal visibility on the Hokuetsu-Hoku Line, those showing the problem related to equipment or the learning of drivers were excluded. Then, using the data, the effect of aspect type and train speed was checked for each driver and signal type. As far as the signal visibility (the distance of signal calling) is concerned, the result shows that the speed-up signal is equivalent to or more than the proceed signal while the repeating speed-up signal is equivalent to the repeating proceed signal. Difference due to the train speed range was not observed as a whole concerning the work allowance (variance of the distance of calling) and there was no sign of possible damage of high-speed running to the work allowance.

As so far described, it was confirmed that the use of speed-up signal and train operation at maximum $160 \mathrm{~km} / \mathrm{h}$ on the Hokuetsu-Hoku Line would present no problem in terms of the signal visibility and work allowance.

The result of the signal visibility test described in this paper has contributed to authorization of train speed of $160 \mathrm{~km} / \mathrm{h}$ and approval of the signaling mechanism with speed-up signal for the Hokuetsu-Hoku Line. Operation of the high-speed train was also authorized in "the Special Handling of Railway Operation Rules". With this achievement, we are confident that speed-up of the local line by means of wayside signaling has made an important step forward.

\section{References}

1) Ogawa, Y., Yoneyama, S., Ona, K.: "A Study on the Visibility of the Railroad Signals," Bulletin of Railway Labour Sci. Res. Inst. (in Japanese), 40, pp.125-132, 1986

2) Ugajin, H., Tamura, T., Yoneyama, S., Fujinami, K.: "Some New Signal Aspects for High-Speed Train on Wayside Signaling," RTRI Report (in Japanese ), 9, 10, pp.4954,1995 